\title{
Calorimetric Non-Destructive Assay of Large Volume and Heterogeneous Radioactive Waste Drums
}

\author{
W. R. Kubinski ${ }^{1}$, C. Carasco, D. Kikola ${ }^{1}$, C. Mathonat, D. Ricard ${ }^{4}$, \\ D. Tefelski ${ }^{1}$, H.Tietze-Jaensch ${ }^{5}$
}

\author{
${ }^{1}$ Warsaw University of Technology, Plac Politechniki 1, Warszawa 00-661, Poland \\ ${ }^{2}$ CEA/DEN/DTN Cadarache, F-13108 Saint-Paul-Lez-Durance, France \\ ${ }^{3}$ KEP Technologies, Rue de l'Oratoire 7, 69300 Caluire et Cuire, France \\ ${ }^{4}$ Andra, 1/7 rue Jean Monnet, Parc de la Croix-Blanche, F-92298 Châtenay-Malabry, France \\ ${ }^{5}$ Forschungszentrum Jülich, Wilhelm Johnen Strasse, Jülich 52428, Germany
}

\begin{abstract}
The EU-CHANCE project aims at the issue of the characterization of conditioned radioactive waste (CRW) and one objective of CHANCE is to focus on: Calorimetry as a comprising non-destructive technique to reduce uncertainties on the inventory of radwaste containing shielded and hidden material difficult to be measured by other means.

A MCNP6-based numerical study comprising the particle flux out of a 200L mock-up drum in a Large Volume Calorimeter (LVC) currently manufactured by KEP Nuclear (France) will be presented and discussed. For the analyses, the particle flux and energy deposition in each layer of the calorimeter were determined. The results yield that a significant fraction of the radiation would leave the system and not contribute to the measurable heat deposition. The expected energy deposition is obtained and cumulated for each layer over the whole energy range revealing the fraction of particles actually escaping the LVC calorimeter. While this escape fraction needs and can be determined, the LVC is a very suitable apparatus for the anticipated experiments on large and heterogeneous waste drums that possibly contain deeply buried beta-emitters (e.g. Sr/Y-90) or shielded alpha-sources hidden inside the drum with a significant level of gamma and neutron radiation background radiation. The high-energy part of this gamma and neutron flux may even reach the reference chamber of the calorimeter and deposit some energy there, compromising the calibration and may cause a double-bias.
\end{abstract}

\section{INTRODUCTION}

$\mathrm{I}^{\mathrm{N}}$ $\mathrm{N}$ order to manage, securely store and efficiently process radioactive materials, their precise characterization is essential and mandatory. However, conditioned waste, unlike raw materials, is sealed and embedded in a solid material matrix making access difficult. In addition, radioactive waste may contain materials of various origin, and the spectrum of their radiation can be quite complex. Often waste drums, in particular after years of interim storage, are no more in their state of initial state, thus unfit for final disposal. The comprehensive characterisation of radioactive waste is a requisite for its safe final disposal, and it is often quite complicated. It requires a set of specific assessment techniques, preferably non-destructive methods.

Amongst others calorimetry is one of the methods for the determination of nuclear material masses (or activities) by the quantitative measurement of heat flux, emerging from a sample. Calorimetry is used for non-destructive assay (NDA) which means that no sample needs be tempered with or destroyed by the analysis. Heat-flow calorimeters have been used successively for measuring a wide range of thermal powers i.e. from $0.5 \mathrm{~mW}(0.2 \mathrm{~g}$ of low-burnup plutonium) up to $1 \mathrm{~kW}$ for samples varying in size from about $2.5 \mathrm{~cm}$ up to 60 $\mathrm{cm}$ wide and $100 \mathrm{~cm}$ long [3].

As a non-destructive method for the quantitative measuring of the heat emission, calorimetry is used to determine the masses/activities of nuclear material hidden inside nuclear waste compounds. In combination with gamma and neutron emission measurement, calorimetry is well suited for the multi-nuclide assay of conditioned waste drums of largevolumes and possibly heterogeneous content,

All radioactive decay energy converts into heat, eventually. On the one hand, calorimetry encompasses all the radionuclides contained in a sample and emitting radiation that converts into heat by the interaction of the emitted particles with surrounding matter. On the other hand, calorimetry is by no means specific for one or the other radionuclide. Moreover, any radiation that escapes from the calorimetric apparatus remains undetected and unaccounted for. To employ this method as a comprehensive and useful tool for the characterization of nuclear waste, it is therefore essential to support calorimetric assay not only with complementary gamma and neutron emission measurements but also with detailed numerical simulation of the experimental set-up. Then, this method can be turned into a viable one, and potential bias can be eliminated by proper instrument 
calibration. None-the-less, a possible reason of diminished accuracy of calorimetry is the energy leakage caused by fugitive gamma and neutron radiation. This radiation is produced by gamma emitters (e.g. ${ }^{60} \mathrm{Co},{ }^{137} \mathrm{Cs}$ ), as an effect of radioactive decay and various nuclear interaction inside the drum, e.g. bremsstrahlung radiation resulting from intense beta-emitters (e.g. ${ }^{90} \mathrm{Sr} /{ }^{90} \mathrm{Y}$ ). As the accuracy of the assay is one of the key elements of reliable measurements, the radiation leakage must be considered as a major source of uncertainty, which is definitely worthwhile to be studied and analysed in detail [5].

At times of comfortable computing power, numerical calculations can mimic the experimental set-up and radiation vs. matter interactions and so support and supplement the uncertainty analysis associated with the radiation leaking from the calorimeter. One of those numerical methods is the Monte Carlo method based on a probabilistic approach and random numbers. It is becoming more and more frequently used in the analysis of nuclear systems because of the possibility of an easy-play studying the parameters, without unjustified simplifications. Nowadays computing power allows us to simulate a full, three-dimensional nuclear system, made of detectors, fuel assemblies and even whole reactor cores as well as material configurations in radioactive waste drums, and providing reliable results at satisfactorily short calculation times.

The studies presented here were undertaken in the frame of the European research and development project

"CHANCE" (Characterization of conditioned nuclear waste for its safe disposal in Europe), which focuses on specific issues of conditioned radioactive waste characterisation. One of the aims of the project is to design and construct a new, improved Large Volume Calorimeter with reduced detection limits and improved accuracy. The goals of this work were to support the instrument's evaluation and validation by numerical calculations and simulation. This study's aim is to set-up an appropriate computational environment and to perform a series of simulations in order to understand and estimate the influence of the radiation leakage on the final results and their precision.

\section{THE MODEL}

Matching the design of the calorimeter, a simplified but still viable numerical model was implemented using MCNP6 code. The numerical model is presented in Figure 1. It consists of different layers, just alike the real KEP-LVC calorimeter. There is a cylindrically shaped sample drum at the centre of the measurement chamber which is filled with air. The next layer is the octagon-shaped structure with the heat flux detectors mounted on each wall. Then, there is the package of homogenization (red layers in Figure 1) and alternating insulation layers (blue layers), up to sixth layer each. Underneath the measurement chamber there is the reference chamber (or ghost chamber) with a phantom aluminium block.

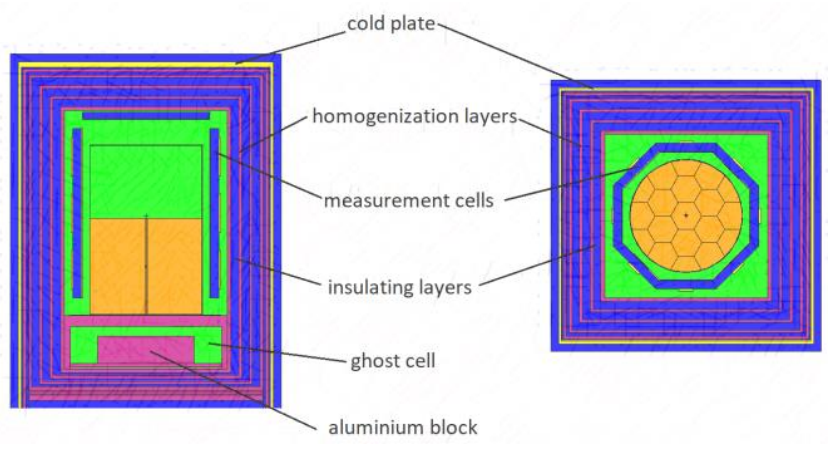

Figure 1. Simplified model of the calorimeter.

\section{CONSIDERED SCENARIOS}

To check how the distribution of radioactive material inside the drum influences the radiation leakage, three scenarios were realised in the simulations. For all the scenarios the drum was filled with a virtual radioactive composition up to $50 \mathrm{~cm}$ (active part of the drum). The remaining part was filled with air.

\section{$1^{\text {st }}$ scenario: most conservative}

The first source configuration, hereafter called the "most conservative" set-up was a drum filled with sand and one pin placed in its centre. In the middle of the pin there was a container with the virtual sampled source. In this scenario it was assumed that all the radioactivity was buried deep inside the drum, thus on average, particles needed the longest possible path to escape the system.

\section{$2^{\text {nd }}$ scenario: homogenous source}

In this scenario, hereafter called the "homogenous" set-up, it was assumed that the virtual radioactive material is uniformly distributed within the whole volume of the active part of the drum. The active part was filled only with sand and particles were sampled inside this whole volume. The amount of escaping radiation was, of course, higher in this case, as the particles could appear nearby the wall of the drum as well.

\section{$3^{\text {rd }}$ scenario: least conservative}

A third kind of source configuration, hereafter called "least conservative" set-up was a drum, similar to case I, but with only one pin filled and this one placed closest to the edge of the drum. In addition, the container with the virtual radioactive material was located at the bottom of the pin. In this scenario the particles, on average, had the shortest pathway to leave the system.
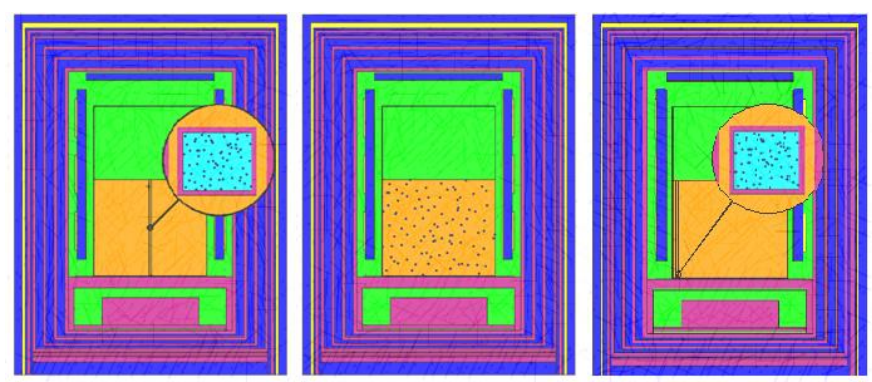

Figure 2. Considered source scenarios. Most conservative (left), homogenous (middle) and least conservative (right). 
Figure 2 shows the differences between sampling particles scenarios in all three kinds of the source configurations. The radioactive composition was modelled by a virtual gamma source and a virtual neutron source at energies from $10 \mathrm{keV}$ up to $10 \mathrm{MeV}$. In addition, some calculations of waste composition for beta and alpha emission were carried out, too, but as expected, no escape radiation has been found.

\section{RESULTS}

\section{A. Particles flux}

In order to understand how the particles behave inside the calorimeter, the particle flux was checked using tally 4 with the mesh option and normalized to $10^{6}$ particles source strength. The flux was calculated on a XY mesh made of 10000 rectangular cells, each $1.4 \mathrm{~cm}$ wide and $188 \mathrm{~cm}$ high. The flux was also determined along $\mathrm{Z}$ axis, on a mesh made of 300 rectangular cells, $72 \mathrm{~cm}$ wide and $0.6 \mathrm{~cm}$ high. The obtained 3D fluxes are illustrated in Figure 3. The fluxes were analysed for the three scenarios and three different source energies $(0.1 \mathrm{MeV}, 1 \mathrm{MeV}$ and $5 \mathrm{MeV})$. For the clarity of the results presented, the charts were zoomed up to the range of $\left(0,10^{-4}\right) \# /$ particle $/ \mathrm{cm}^{2}$.

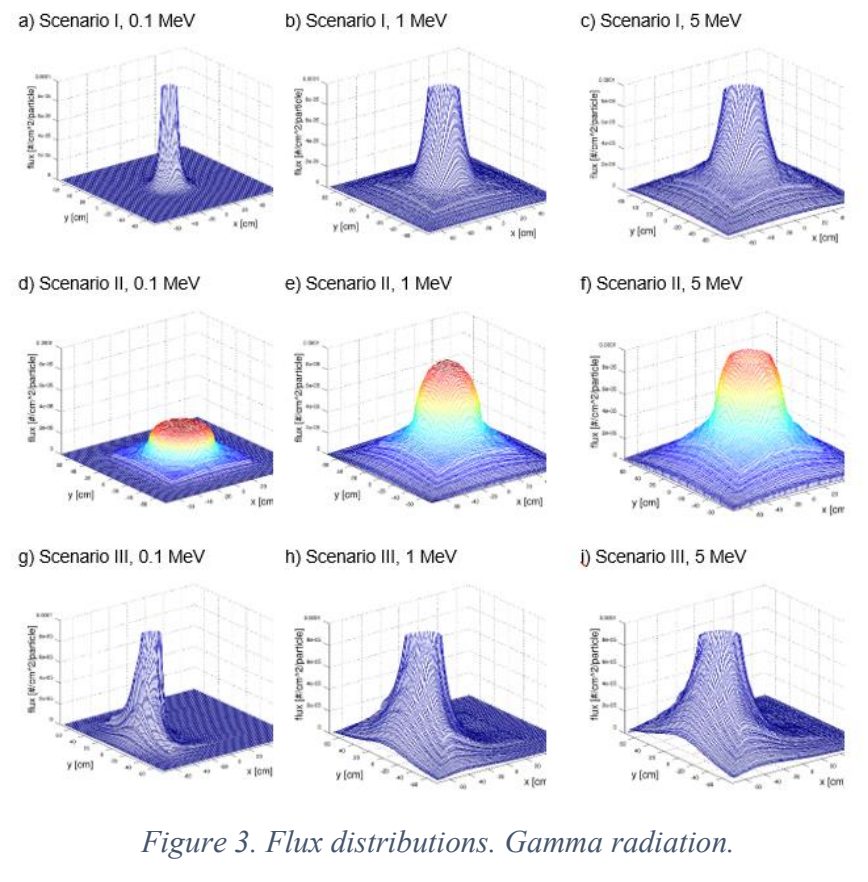

The results confirm that for the $0.1 \mathrm{MeV}$ source almost all the radiation is deposited inside the system. Despite all particles being absorbed and no radiation escaping the system, even for low energy gammas not all the heat is deposited inside the Peltier elements where it is detected. For the homogenous and least conservative scenario, still some layers of the calorimeter are visible, which means that some part of the radiation penetrates past the detection zone and the homogenization and insulation parts. Only the heat deposited inside the drum and measurement parts are detectable, thus not all the total deposited energy can be measured. And for higher energies, certainly even more particles leave the system, a results that has been expected.

The charts (Figure 3) are meant to be just illustrative. To quantify and compare the fluxes, especially the tails which describe the radiation leakage, two-dimensional charts are plotted along the $\mathrm{X}$ and $\mathrm{Z}$ axes. Figure 4 and Table 1 show a juxtaposition of fluxes at mid-energy (i.e. $1 \mathrm{MeV}$ ) for all three scenarios. a)

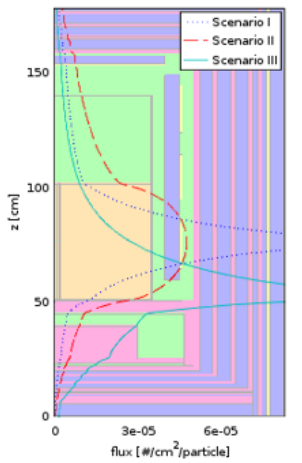

Figure 4. Gamma particles flux. b)

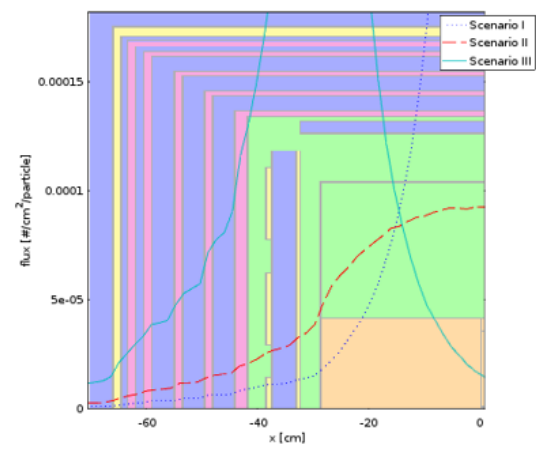

One can see that the energy of the source has significant influence on the flux distribution. The flux plotted along $\mathrm{Z}$ axis (Figure 4a) shows that some part of it reaches into the ghost chamber and beyond. In general, for higher energies, the flux penetrates more layers of the calorimeter, as well as the reference parts which cause inevitably some double bias.

TABLE I

GAMMA FLUX ESCAPE [\#/PARTICLE/CM²]

\begin{tabular}{ccccc}
\hline \hline & \multicolumn{4}{c}{ Escape along Z-axis } \\
\hline scenario: & bottom: & rel. error: & top: & rel. error: \\
\hline I & $1.93 \cdot 10-7$ & $2.7 \%$ & $4.63 \cdot 10-7$ & $1.4 \%$ \\
II & $5.08 \cdot 10-7$ & $1.9 \%$ & $1.60 \cdot 10-6$ & $1.0 \%$ \\
III & $1.76 \cdot 10-6$ & $0.7 \%$ & $2.13 \cdot 10-7$ & $2.1 \%$ \\
& & & & \\
& Flux in the & & escape & \\
& aluminum & & along X- & \\
& block: & rel. error: & axis & rel. error: \\
I & $2.48 \cdot 10-6$ & $0.1 \%$ & $9.47 \cdot 10-7$ & $6.7 \%$ \\
II & $5.82 \cdot 10-6$ & $0.5 \%$ & $2.75 \cdot 10-6$ & $4.3 \%$ \\
III & $2.03 \cdot 10-5$ & $0.4 \%$ & $1.07 \cdot 10-5$ & $2.0 \%$ \\
& & & & \\
\hline \hline
\end{tabular}

The results show that source distribution pattern has a strong influence on the flux distribution. In case of the flux along $\mathrm{X}$-axis (Figure $4 \mathrm{~b}$ ) and the source close to the wall of the drum, the flux leakage is around four times higher than compared to the homogeneous case and around 11 times higher compared to the most conservative scenario I.

\section{B. Energy deposition}

As it was mentioned, not all the heat is likely to be detected by the calorimeter's heat detectors (Peltier elements). In order to fully simulate the actual situation the heat measured by the device, the energy/heat deposition in all the layers was determined. 
TABLE II

ENERGY DEPOSITION IN THE CALORIMETER'S LAYERS HOMOGENOUS $1 \mathrm{MEV}$ GAMMA SOURCE

\begin{tabular}{|c|c|c|}
\hline \multirow{2}{*}{$\begin{array}{l}\text { source energy }[\mathrm{MeV} / \text { particle }] \text { : } \\
\text { Layer: }\end{array}$} & \multicolumn{2}{|l|}{1} \\
\hline & $\begin{array}{l}\text { energy } \\
\text { deposition: }\end{array}$ & $\begin{array}{l}\text { rel. } \\
\text { error: }\end{array}$ \\
\hline thermal block & $6.8 \%$ & $0.1 \%$ \\
\hline insulation layer 1 & $0.0 \%$ & $0.2 \%$ \\
\hline homogenization layer 1 & $4.0 \%$ & $0.2 \%$ \\
\hline insulation layer 2 & $0.0 \%$ & $0.2 \%$ \\
\hline homogenization layer 2 & $3.2 \%$ & $0.2 \%$ \\
\hline insulation layer 3 & $0.0 \%$ & $0.2 \%$ \\
\hline homogenization layer 3 & $2.5 \%$ & $0.2 \%$ \\
\hline insulation layer 4 & $0.0 \%$ & $0.3 \%$ \\
\hline homogenization layer 4 & $2.0 \%$ & $0.2 \%$ \\
\hline insulation layer 5 & $0.0 \%$ & $0.3 \%$ \\
\hline cold plate & $1.7 \%$ & $0.3 \%$ \\
\hline insulation layer 6 & $0.0 \%$ & $0.3 \%$ \\
\hline drum & $(+) 64.5 \%$ & $0.1 \%$ \\
\hline measurement chamber & (+) $\quad 0.0 \%$ & $0.2 \%$ \\
\hline ghost chamber walls & $5.5 \%$ & $0.3 \%$ \\
\hline ghost cell and phantom & (-) $0.5 \%$ & $1.1 \%$ \\
\hline ghost cell reference part & (+) $0.0 \%$ & $0.8 \%$ \\
\hline measurement plates & (+) $1.9 \%$ & $0.2 \%$ \\
\hline octagon structure & $0.0 \%$ & $0.2 \%$ \\
\hline reference plates & (-) $0.4 \%$ & $0.4 \%$ \\
\hline total: & $93.2 \%$ & $0.1 \%$ \\
\hline detected: & $65.5 \%$ & $0.1 \%$ \\
\hline
\end{tabular}

As expected, for the uniformly distributed radioactive material, the radiation leakage increases and not all the heat is detected. Additional simulations showed that even in the case of low energy gamma radiation (i.e. $100 \mathrm{keV}$ ), and despite almost $100 \%$ of the energy being deposited inside the system, more radiation penetrates the layers and only around $83 \%$ of the energy would be detected. For higher energies half of the energy may escape from being detected.

\section{SUMMARY}

The results showed that some part of gamma radiation of energies $>100 \mathrm{keV}$ is not deposited inside the LVC calorimeter, this especially, for the scenarios of the radiation source being placed nearby the edge of the drum (i.e. homogenous and least conservative sources). Despite a good part of the energy is deposited inside calorimeter measurement chamber, some gamma radiation penetrates the outer layers and escaped the deposited energy detection. Some other part of radiation may also be deposited inside the reference parts of the calorimeter and cause a double-bias.. In order to summarise and create an indicator for the future measurements, the simulated total errors of the measurement for each of the scenarios at different energies are presented in Figure 5. Bolded energies will contribute to the calorimeter's response. These marked with a plus $(+)$ increase the final result, those with a minus (-) reduce it. Remining deposition do not significantly affect the final response.

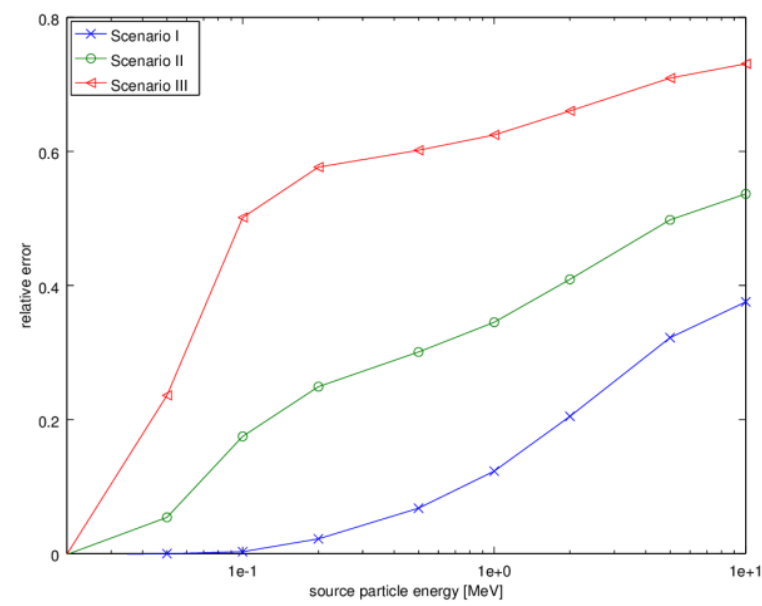

Figure 5. Maximal share of missed heat, gamma radiation.

One can see that the total error of the measurement, connected with the penetrative character of the gamma radiation, is more or less proportional to logarithm of the source energy. As for the energy deposition and detection probability, the results suggest that even for a $50 \mathrm{keV}$ gamma source placed close to the edge of the drum around $24 \%$ of the emitted energy cannot be detected. For the least conservative scenario at the highest simulated energy, even around $73 \%$ escape detection.

\section{CONCLUSION}

The result showed that the radiation leakage has a significant influence on the heat flux measurement, namely for the high energy gamma and neutron particles. Even if the energy of the radiation is not high enough to leave the system, the penetrative character of the radiation impacts the accuracy of the measurement by heat deposition inside the reference parts and outside the detector sphere. The accuracy is being compromised up to $\sim 50 \%$ because of the unwanted and undesirable outside deposition of both, neutrons and photons. In general, it is fair to say that the radiation leakage is a complicated problem, and for some isotopes and configurations it can either impair the precision of the heat measurement or have no influence at all. Therefore, and especially for radioactive waste characterization, it is an interesting issue worthwhile further analyses, measurements and simulations. It also provides the chance to improve or at least understand the processes which cause the uncertainties of calorimetric assay, which is one of the main goals of the CHANCE project.

The model is implemented and works properly. It can be used as a tool for the uncertainties assessment support or can to indicate the outcome of any measurements anticipated. Therefore, the model will certainly be used again and may be 
modified as to adjust it to the real experiments, by changing the drum composition, the embedding material matrix, the energy spectrum and source location. In addition, the model can also be used to improve the existing LVC construction by adding some extra radiation shielding structures or filling the measurement chamber with a proper material to decrease the influence of the radiation leakage.

\section{REFERENCES}

[1] Status and Trends in Spent Fuel and Radioactive Waste Management, IAEA Nuclear Energy Series, No. NW-T-1.14, 2018 https://www-pub.iaea.org/MTCD/Publications/PDF/P1799_web.pdf [2] The CHANCE project "Characterization of conditioned nuclear waste for its safe disposal in Europe". DEM 2018 - Dismantling Challenges: Industrial Reality, Prospects and Feedback Experience, D. Ricard, D., Plumeri, S., Boucher, L., Rizzo, A., Tietze-Jaensch, H., Mathonat, C., Bruggeman, C., Velthuis,J., Thompson, L., Genoud, G., Bucur, C., Kikola, D., Zakrzewska-Koltuniewicz, G France, Avignon - 2018 and CHANCE project official website, http://chance-h2020.eu, accessed on 05.08.2018

[3] Principles and applications of calorimetric assay, D.S. Bracken, C.R. Rudy, Los Alamos Report LA-UR-07-5226, 2007, https://www.lanl.gov/orgs/n/n1/panda/10.\%20Calorimetry.pdf

[4] Deliverable report D3.1 Applicability of Calorimetry to Real Waste Characterisation, CHANCE project deliverable, C. Carasco, B. Perot et. al., 2018, publ. on CHANCE 2020: http://chance-h2020.eu

[5] Application Guide to Safeguards Calorimetry, D.S. Bracken, R.S. Biddle et. al., LA-13867-M, January 2002

[6] Performance values for non-destructive assay (NDA) techniques applied to safeguards: The 2002 evaluation by Esarda, P. Schwalbach, M.T.Swinhoe, ResearchGate, 2676830081, 2002

[7] NDA quantification of Nuclear Waste Containers, G. Jossens, P. Girones, C. Mathonat, A. Godot, ResearchGate, 278025167, July 2015

[8] https://www.nucleonica.com, accessed on 30.08.2018

[9] Initial MCNP6 Release Overview - MCNP6 version 1.0, J. T. Goorley, M. R James, et. al., LA-UR-13-22934, April 2013

[10] Development of a New Monte Carlo Reactor Physics Code, Jaakko Leppänen, VTT PUBLICATIONS 640, June 2007

[11] https://www.researchgate.net/figure/Flow-diagram-to-show-thesimplified-transport-process-for-a-single-neutron-historyin_fig7_277027129, accessed on 30.08.2018

[12] An MCNP primer, J. K. Shultis, R. E. Faw, Kansas State University, 2011, https://www.mne.k-state.edu/ jks/MCNPprmr.pdf [13] Expressing Precision and Bias in Calorimetry, D.K. Hauck, S. Croft, D.S. Bracken, NM 87545, 2010

[14] https://www-nds.iaea.org/exfor/endf.htm accessed on 05.09.2018 